Published in final edited form as:

Eur Urol. 2014 July ; 66(1): 77-84. doi:10.1016/j.eururo.2014.02.035.

\title{
ClearCode34: A Prognostic Risk Predictor for Localized Clear Cell Renal Cell Carcinoma
}

\author{
Samira A. Brooks ${ }^{a}$, A. Rose Brannon ${ }^{a, b}$, Joel S. Parker ${ }^{a}$, Jennifer C. Fisher ${ }^{a}$, Oishee Sen ${ }^{a}$, \\ Michael W. Kattan ${ }^{c}$, A. Ari Hakimi ${ }^{d}$, James J. Hsieh ${ }^{d}$, Toni K. Choueiri ${ }^{e}$, Pheroze Tambolif \\ Jodi K. Maranchie ${ }^{g}$, Peter Hinds ${ }^{g}$, C. Ryan Miller ${ }^{a, h}$, Matthew E. Nielsen ${ }^{a, i, j}$, and W. Kimryn \\ Rathmella,k, \\ aUNC Lineberger Cancer Center, Chapel Hill, NC, USA \\ bMemorial Sloan Kettering Cancer Center, New York, NY, USA \\ 'Department of Quantitative Health Sciences, Cleveland Clinic, Cleveland, OH, USA \\ dDepartment of Surgery, Urology Service, Memorial Sloan Kettering Cancer Center, New York, \\ USA \\ eDepartment of Medical Oncology and Kidney Cancer Center, Dana Farber Cancer Institute, \\ Boston, MA, USA \\ fDepartment of Pathology, MD Anderson Cancer Center, Houston, TX, USA \\ 9Department of Urologic Oncology, University of Pittsburgh Medical Center, Pittsburgh, PA, USA \\ hDepartment of Pathology, University of North Carolina at Chapel Hill, Chapel Hill, NC, USA \\ 'Department of Urology, University of North Carolina at Chapel Hill, Chapel Hill, NC, USA \\ iDepartment of Epidemiology, University of North Carolina at Chapel Hill, Chapel Hill, NC, USA \\ ${ }^{k}$ Department of Medicine, Division of Hematology and Oncology, and Department of Genetics, \\ University of North Carolina at Chapel Hill, Chapel Hill, NC, USA
}

\footnotetext{
(C) 2014 European Association of Urology. Published by Elsevier B.V. All rights reserved.

*Corresponding author. 450 West Drive, CB 7295, UNC Lineberger Cancer Center, Chapel Hill, NC, 27599, USA. Tel.: +1 919 966 8644; Fax:+1919966 8212. Rathmell@med.unc.edu (W.K. Rathmell).

Publisher's Disclaimer: This is a PDF file of an unedited manuscript that has been accepted for publication. As a service to our customers we are providing this early version of the manuscript. The manuscript will undergo copyediting, typesetting, and review of the resulting proof before it is published in its final citable form. Please note that during the production process errors may be discovered which could affect the content, and all legal disclaimers that apply to the journal pertain.

Author contributions: W. Kimryn Rathmell had full access to all the data in the study and takes responsibility for the integrity of the data and the accuracy of the data analysis. Study concept and design: Brooks, Brannon, Parker, Nielsen, Rathmell.

Acquisition of data: Brooks, Brannon, Hakimi, Hsieh, Choueiri, Tamboli, Maranchie, Hinds, Rathmell.

Analysis and interpretation of data: Brooks, Brannon, Parker, Nielsen, Rathmell.

Drafting of the manuscript: Brooks, Nielsen, Rathmell.

Critical revision of the manuscript for important intellectual content: Brooks, Brannon, Parker, Kattan, Hakimi, Hsieh, Choueiri,

Tamboli, Maranchie, Hinds, Nielsen, Rathmell.

Statistical analysis: Brooks, Brannon, Parker, Kattan, Nielsen.

Obtaining funding: Rathmell.

Administrative, technical, or material support: Fisher, Sen.

Supervision: Rathmell.

Other (specify): None.
} 


\section{Abstract}

Background-Gene expression signatures have proven to be useful tools in many cancers to identify distinct subtypes of disease based on molecular features that drive pathogenesis, and to aid in predicting clinical outcomes. However, there are no current signatures for kidney cancer that are applicable in a clinical setting.

Objective-To generate a signature biomarker for the clear cell renal cell carcinoma (ccRCC) good risk (ccA) and poor risk (ccB) subtype classification that could be readily applied to clinical samples to develop an integrated model for biologically defined risk stratification.

Design, setting, and participants-A set of 72 ccRCC sample standards was used to develop a 34-gene classifier (ClearCode34) for assigning ccRCC tumors to subtypes. The classifier was applied to RNA-sequencing data from 380 nonmetastatic ccRCC samples from the Cancer Genome Atlas (TCGA), and to 157 formalin-fixed clinical samples collected at the University of North Carolina.

Outcome measurements and statistical analysis-Kaplan-Meier analyses were performed on the individual cohorts to calculate recurrence-free survival (RFS), cancer-specific survival (CSS), and overall survival (OS). Training and test sets were randomly selected from the combined cohorts to assemble a risk prediction model for disease recurrence.

Results and limitations-The subtypes were significantly associated with RFS ( $p<0.01$ ), CSS $(p<0.01)$, and OS $(p<0.01)$. Hazard ratios for subtype classification were similar to those of stage and grade in association with recurrence risk, and remained significant in multivariate analyses. An integrated molecular/clinical model for RFS to assign patients to risk groups was able to accurately predict CSS above established, clinical risk-prediction algorithms.

Conclusions-The ClearCode34-based model provides prognostic stratification that improves upon established algorithms to assess risk for recurrence and death for nonmetastatic ccRCC patients.

Patient summary-We developed a 34-gene subtype predictor to classify clear cell renal cell carcinoma tumors according to $\mathrm{ccA}$ or $\mathrm{ccB}$ subtypes and built a subtype-inclusive model to analyze patient survival outcomes.

\section{Keywords}

Biomarker; ccRCC; Kidney cancer; Renal cell carcinoma; TCGA; Prognosis

\section{Introduction}

The majority of renal cell carcinoma (RCC) or kidney cancer patients have the clear cell RCC (ccRCC) subtype. Although extensive effort has been devoted to identifying molecular biomarkers for RCC, there are few validated markers that aid disease prognosis, and none are used routinely in clinical practice [1-6]. Thus, transcriptional biomarkers present a potentially target-rich environment toward the goal of improving our understanding of underlying ccRCC biology. 
Recently, we identified two subtypes of clear cell RCC, ccA and ccB, based on patterns of differential gene expression, that revealed distinct biologic signatures [7]. These subtypes appear to provide prognostic information, with tumors classified as ccA associated with significantly better survival compared to $\mathrm{ccB}$ in a retrospective cohort [7]. The $\mathrm{ccA} / \mathrm{ccB}$ classification was subsequently validated in a meta-analysis of $480 \mathrm{ccRCC}$ tumors, suggesting this profile may have value for risk stratification [8].

Building on this foundation, in the present study we demonstrate the utility of a novel tool to identify the ccA and ccB groups in ccRCC. This molecular tool comprises a 34-gene expression signature (ClearCode34) and an accompanying protocol for $\mathrm{ccA} / \mathrm{ccB}$ classification. Clinical utility of the classifier is demonstrated by (1) accurate and reproducible classification of ccRCC tumors into ccA and ccB molecular subtypes, (2) validation of prognostic classification, (3) adaptation to clinical samples in the form of formalin-fixed paraffin-embedded (FFPE) tissues, and (4) constructing a more precise unified model of ccRCC subtype and standard clinical variables to assign individual ccRCC patients into clinically informative risk categories.

\section{Patients and methods}

\subsection{Patients and clinical samples}

The ccRCC samples $(n=95)$ previously analyzed by gene expression microarray were clustered to define the ccA and ccB classifications [7]. Of these, 72 were chosen as references to develop the 34-gene panel based on concordant subtype classifications determined by two methods: logical analysis of data and ConsensusCluster [7-9] (Fig. 1a).

Prognostic assessment of ClearCode34 was performed using RNA-sequence data of the Cancer Genome Atlas (TCGA). Median follow-up for this cohort was 38 mo (range: 0-113 mo), with seven patients having no follow-up. Clinical data (last modified August 23, 2013) were downloaded from TCGA Data Portal [10]. Expert members of TCGA Analysis Working Group performed pathologic re-evaluation, and cases not definitively representing clear cell histology were excluded from further analysis [11]. Recurrence and survival data were taken from TCGA Biotabs database, with appropriate permissions, with supplementation by the clinical TCGA working group database (version dated April 11, 2013) [10].

Clinical utility of ClearCode34 was performed using randomly selected specimens collected between 1992 and 2010 at the University of North Carolina (UNC) from patients with nonmetastatic ccRCC, and stored in the pathology archive as formalin-fixed paraffinembedded (FFPE) blocks. Median follow-up for this UNC cohort was 54 mo (range: 3-232 mo). Stage was reclassified using the American Joint Committee on Cancer's Cancer Staging Manual, 7th edition (AJCC-7) for all cases preceding 2010 and an expert genitourinary oncologist and an expert surgical pathologist verified clinical and pathologic variables.

Only patients with nonmetastatic disease at the time of nephrectomy were used for the study. This did include a small number of patients with T4 lesions and who had extensive local 
disease classified by AJCC-7 as stage IV but M0 with regard to distant metastasis. No patients received systemic therapy for ccRCC before nephrectomy or prior to clinical recurrence. All samples and data were obtained with appropriate institutional review board approvals.

\subsection{The Cancer Genome Atlas data analysis}

TCGA RNA sequence data were normalized to the upper quartile of normal counts. For analysis, the data were log-transformed (base 2) and genes were median centered.

\subsection{Formalin-fixed paraffin-embedded sample preparation}

UNC cohort FFPE samples were sliced (5-7 $\mu \mathrm{m}$ thick) onto slides or prepared as scrolls 10$20 \mu \mathrm{m}$ thick. The surface area of the tissue sectioned was a minimum of $1 \mathrm{~cm}^{2}$. Xylene was added and washed twice with $100 \%$ ethanol. Pellets were suspended in $10 \mathrm{mM} 2-(\mathrm{N}-$ morpholino) ethanesulfonic acid pH 6.5 or Proteinase K digest buffer (Qiagen Gaithersburg Inc, Gaithersburg, MD, USA) with $0.5 \%$ SDS and $5 \mu$ Proteinase K $(20 \mathrm{mg} / \mathrm{ml})$.

Suspensions were incubated $\left(55^{\circ} \mathrm{C}\right)$, Proteinase $\mathrm{K}$ inactivated $\left(80^{\circ} \mathrm{C}\right)$, and supernatant collected.

\subsection{NanoString analysis}

The UNC genomics core processed $5 \mu 1$ lysate or $100 \mathrm{ng}$ RNA for hybridization against NanoString probes (NanoString Technologies Inc, Seattle, WA, USA) [12], posthybridization in the nCounter Prep Station, and data collection with the nCounter digital analyzer (NanoString Technologies Inc, Seattle, WA, USA). Sample-specific background was subtracted using values from included negative controls. Data were normalized using the geometric mean of housekeeping genes and $\log$ transformed (base 2). See the Supplement for methods details.

\subsection{Development of a gene expression classifier of ccA or ccB}

To develop a minimal gene set classifier for assigning ccA or ccB subtype, prediction analysis of microarray (PAM) [13], a centroid-based classification algorithm, was applied to the microarray reference data (Fig. 1). PAM was used due to its reproducibility in subtype classification compared to other centroid-based prediction methods [14]. We used a list of genes (Supplemental Table 1) that encompassed those previously associated with ccA/ccB classification [7], genes differentially expressed between the ccA/ccB subtypes using significance analysis of microarrays, and other published markers [2-6]. Ninety-four percent of the tumors were classified correctly (68 of 72) using a PAM model of 34 genes (Table 1) based on nearest centroids. This gene list was labeled ClearCode 34.

Cross-validation (random 10\% left out in each of 50 cycles) was applied to the microarray reference set to evaluate the accuracy of the classifier and anticipated performance on independent sample cohorts. Unsupervised clustering and ConsensusCluster [9] were used to further assess assignment accuracy by the minimized gene panel in the microarray standard set by comparing PAM-derived subtype assignments with those determined previously [7]. 


\subsection{Statistical analysis}

All continuous variables were described with the median and range values. Recurrence, or relapse, was defined as the date from nephrectomy to the date that recurrence or metastasis was detected by imaging or pathology report. Cancer-specific survival (CSS) was defined as the time from nephrectomy to death resulting specifically from ccRCC; patients who remained alive, died of other reasons, or had unknown causes of death were censored for this outcome at the date of last follow-up or death. Overall survival (OS) was defined as the time from the nephrectomy to death of any cause. The probability of death or recurrence was determined by using the Kaplan-Meier method, with log-rank tests assessing the differences between the groups. CSS was analyzed using the competing risk method (cmprsk $\mathrm{R}$ package; R Project for Statistical Computing, Vienna, Austria). Cox proportional hazard models and likelihood ratio using OS, CSS, and recurrence outcomes were used to compare competing survival models. ccRCC subtype, Fuhrman grade, and stage were modeled as additive predictors of outcome. Cox proportional hazard models were used to estimate hazard ratios (HRs) and 95\% confidence intervals (CIs).

\subsection{Developing a relapse risk model}

The TCGA and UNC cohorts were combined to develop a risk model for tumor relapse (Fig. 2). The combined cohort consisted of 531 patients, after removing six patients with missing grade information. The combined cohort was randomly split into two sets of equal size for use as training and evaluation cohorts for the prognostic model. Samples were used only once for either the training or test set. A multivariable Cox model with ccA/ccB subtype, stage, and Fuhrman grade as additive terms was fit with Ridge regression. Tenfold crossvalidation was performed in the training set to optimize the penalty parameter. A final model was fit to the entire training set using the optimized parameters and then applied to the test set. See the Supplement for details in identifying risk groups and assigning cases.

\section{Results}

\subsection{Subtype comparison for prognosis and recurrence in the Cancer Genome Atlas data set}

To evaluate the prognostic utility of ClearCode 34 for ccA and ccB ccRCC tumors, KaplanMeier analysis was used to assess tumor recurrence and survival rates by subtype assignment in tumor samples from 380 nonmetastatic ccRCC patients from the TCGA data set (Table 2). Univariate analysis revealed ccB patients experienced tumor recurrence earlier and more frequently than ccA (HR: 2.3; 95\% CI, 1.6-3.3; $p=4.3 \times 10^{-6}$ ) (Fig. 3a). Moreover, ccB patients had almost three times the risk of death from disease (HR, 2.9; $95 \%$ CI, 1.6-5.6; $p=0.0005$ ) and more than two times the risk of death from any cause compared to $\mathrm{ccA}$ (HR: $2.4 ; 95 \% \mathrm{CI}, 1.6-3.7 ; p=2.3 \times 10^{-5}$ ) (Fig. $3 \mathrm{~b}$ and $3 \mathrm{c}$ ). Competing risk analysis further validated the differences in survival between the subtypes, which showed their specificity as prognostic tools for ccRCC (CSS, $p=0.002$; OS, $p=0.037$ ). 


\subsection{Biomarker validation in an independent cohort}

Since ClearCode34 demonstrated prognostic value in the TCGA cohort from known clinical samples, we next validated the classifier in an independent group of clinical specimens using the NanoString platform. Applying the classifier to a cohort of 157 nonmetastatic ccRCC, archived FFPE samples (Table 3), 69 samples were assigned as ccA subtype and 88 as ccB, and, again, the subtype classifications followed survival patterns seen previously (Fig. 4). In this cohort, ccB cases experienced tumor relapse after nephrectomy more frequently (HR: 2.1; 95\% CI, 1.3-3.4; $p=0.001$ ) (Fig. 4a) and had higher risk of both cancer-specific mortality (HR: 3.0) (Fig 4b) and overall mortality (HR: 2.2) (Fig. 4c) compared to patients classified as ccA. In addition, cancer-specific deaths $(p=0.013)$, but not overall deaths $(p=$ 0.344), remained significant between subtypes after competing risk analysis.

\subsection{Prognostic risk models for recurrence in patients with nonmetastatic clear cell renal cell carcinoma}

We next sought to determine if a recurrence risk model encompassing both ClearCode 34 ccRCC subclassification along with stage and Fuhrman grade could be used to enhance the assessment of patient risk. A Cox model for recurrence was assembled using a combined cohort including both the TCGA and UNC cohorts. In the final model, subtype classification ( $p=0.0009$ ), Fuhrman grade (I/II vs greater; $p<0.0001$ ), and stage (stage I vs greater; $p=$ 0.0007 ) were found to be significant independent variables for predicting recurrence-free survival (RFS) (Supplemental Table 2). Likelihood ratios verified that subtype status was a prognostic factor even after adjustment for stage and Fuhrman grade. A training set was randomly selected from the combined cohort and used to train the risk prediction model, focusing on recurrence. Thresholds for identifying patients for high probability of recurrence within $5 \mathrm{yr}$ following surgical resection were determined by Ridge regression, fit to the training set, and used to stratify low-, intermediate-, and high-risk groups of relapse ( $p=$ $\left.3.04 \times 10^{-9}\right)$ (Fig. 5a). The high- and intermediate-risk groups had a median time to recurrence of 23 and $85 \mathrm{mo}$, respectively. A 50\% survival probability failed to be reached by the low-risk group. Similarly, the three risk groups showed similar survival trends for cancer-specific death after applying the thresholds determined for RFS $\left(p=2.03 \times 10^{-8}\right)$ (Fig. 5b).

Furthermore, we compared our risk assessment tool with existing clinical nomograms to predict death from ccRCC, using multivariate and co-occurrence index (C-index) analysis (Fig. 5c-5f). Our analyses show superiority in assessing risk of ccRCC death by $\mathrm{C}$-index analysis using prognostic classification (ClearCode34 model) compared to the University of California, Los Angeles (UCLA) Integrated Staging System (UISS) [15] and the Mayo Clinic Stage, Size, Grade, and Necrosis (SSIGN) score [16]. Not only did our model better predict disease-specific events (Fig. 5c and 5e), but it also was additive independently of both UISS (UISS, model) and SSIGN (SSIGN, model) (Fig. 5d and 5f), indicating added prognostic information for disease-specific outcomes. Interestingly, subtype classification alone outperformed the UISS algorithm (data not shown). Thus, using ClearCode34 enhances risk stratification, which may guide future clinical planning regarding patient surveillance and adjuvant therapy. 


\section{Discussion}

We developed ClearCode34 to adapt the $\mathrm{ccA} / \mathrm{ccB}$ ccRCC classification to the real world of clinical practice. In this study, we used NanoString to measure expression profiles in clinical specimens. This modality is an emerging, highly reproducible, and low-cost technology for gene expression biomarkers. This work demonstrated that molecular phenotyping using ClearCode 34 has added value to predicting risk above standard clinical and pathologic variables as well as standard ccRCC risk algorithms and may, thereby, enhance patienttreatment planning.

TCGA cohort allowed testing of the prognostic value of ClearCode34 in an independent data set, revealing that $\mathrm{ccA}$ and $\mathrm{ccB}$ were significantly associated with multiple survival end points, which was also observed in the UNC cohort derived from FFPE tissues. We pooled TCGA and UNC cohorts to build a risk predictive model that captures the time period during which the majority of recurrences occur. There remains a potential failure to capture late recurrences, but these tend to be uncommon and typically have a more favorable outcome $[17,18]$. Multivariate analysis for risk of tumor recurrence suggested that the risk prediction model including subtype, stage, and Fuhrman grade provided the best fit given the available variables. Moreover, the model was shown to be a better predictor for CSS than the UISS and SSIGN score, demonstrating the proficiency of this molecular model over standard clinical algorithms. Furthermore, performance status, a key component of the UISS algorithm, is highly subjective, and rarely reported in clinical records. In addition, necrosis is only available upon gross examination and cannot be accurately assessed on biopsy specimens. Although this study did not examine biopsy specimens, we have observed that this biomarker can be applied to biopsy tissue, which could be used to provide essential presurgical risk information. Based on a 2012 study exploring genetic heterogeneity [19], we must be prepared to consider that primary tumors may be genetically divergent from metastases, which may present a challenge in developing tools for predicting the behavior of metastatic disease.

The primary value of this proposed classification system would be for clinicians caring for patients who present with nonmetastatic tumors, seeking guidance regarding postsurgical management, especially as adjuvant therapy is emerging. Although surveillance guidelines exist following nephrectomy, the extent of monitoring is applied in a variable fashion. The availability of an integrated risk-prediction tool may allow guideline refinement for more uniform patient management. Stage and grade remain the most highly significant predictors of risk, given the exceptionally low risk of metastasis with T1a disease, and the exceptionally high risk with T3b/T4 stage. However, it is important to risk stratify patients with intermediate stage tumors, which encompasses an enormous range of both local tumor features and risk.

Gene expression-based tools are widely used in the classification, risk assessment, and therapeutic selection of diseases, such as breast cancers [14,20,21], and are becoming standard for the classification of diffuse large B-cell lymphoma [22] as well as colon cancer [23,24]. Other expression-based systems have examined ccRCC and have demonstrated patterns associated with risk [25]. Because several individual transcripts had previously been 
associated with risk in ccRCC [1-6], we specifically incorporated those features in our model and many were included in the final ClearCode 34 code set, validating their relevance in ccRCC risk prediction. Future prospective studies, with large cohorts of patients, will be needed to fully refine the integrated prognostic algorithm.

\section{Conclusions}

This work presents a novel integration of molecular profiling with standard clinical features to significantly enhance prognostication in ccRCC, thus defining the subset of patients at greatest risk for recurrence for risk-stratified patient care.

\section{Supplementary Material}

Refer to Web version on PubMed Central for supplementary material.

\section{Acknowledgments}

The authors would like to thank the UNC Lineberger Tissue Procurement Facility, Translational Pathology Laboratory, and Genomics Core Facility, other contributors to the Cancer Genome Atlas project, and Mithat Gonen for biostatistical assistance. This paper is subject to the NIH Public Access Policy.

Financial disclosures: W. Kimryn Rathmell certifies that all conflicts of interest, including specific financial interests and relationships and affiliations relevant to the subject matter or materials discussed in the manuscript (eg, employment/affiliation, grants or funding, consultancies, honoraria, stock ownership or options, expert testimony, royalties, or patents filed, received, or pending), are the following: J.S. Parker has consulted for NanoString Technologies, and W.K. Rathmell has a patent filed on the ClearCode test.

Funding/Support and role of the sponsor: This work was supported by a Landon American Association for Cancer Research INNOVATOR grant to W.K. Rathmell; National Research Service Award T32 ES007126 from the National Institute of Environmental Health Sciences to S. Brooks; the Howard Hughes Medical Institute Translational Medicine Fellowship to S. Brooks; the National Cancer Institute of the National Institutes of Health (NIH) Award 5T32CA160001 to A.R. Brannon; the University Cancer Research Fund of the University of North Carolina (UNC) Lineberger Comprehensive Cancer Center to M.E. Nielsen; and the Urology Care Foundation/ Astellas Rising Stars in Urology Research Award to M.E. Nielsen.

\section{References}

[1]. Vasudev NS, Selby PJ, Banks RE. Renal cancer biomarkers: the promise of personalized care. BMC Med. 2012; 10:112. [PubMed: 23016578]

[2]. Ellis LM. The role of neuropilins in cancer. Mol Cancer Ther. 2006; 5:1099-107. [PubMed: 16731741]

[3]. Goel S, Duda DG, Xu L, et al. Normalization of the vasculature for treatment of cancer and other diseases. Physiol Rev. 2011; 91:1071-121. [PubMed: 21742796]

[4]. Harris AL. Hypoxia—a key regulatory factor in tumour growth. Nat Rev Cancer. 2002; 2:38-47. [PubMed: 11902584]

[5]. Wright TM, Brannon A, Gordon J, et al. Ror2, a developmentally regulated kinase, promotes tumor growth potential in renal cell carcinoma. Oncogene. 2009; 28:2513-23. [PubMed: 19448672]

[6]. Yao M, Huang Y, Shioi K, et al. A three-gene expression signature model to predict clinical outcome of clear cell renal carcinoma. Cancer. 2008; 123:1126-32.

[7]. Brannon AR, Reddy A, Seiler M, et al. Molecular stratification of clear cell renal cell carcinoma by consensus clustering reveals distinct subtypes and survival patterns. Genes Cancer. 2010; 1:152-63. [PubMed: 20871783] 
[8]. Brannon AR, Haake SM, Hacker KE, et al. Meta-analysis of clear cell renal cell carcinoma gene expression defines a variant subgroup and identifies gender influences on tumor biology. Eur Urol. 2012; 61:258-68. [PubMed: 22030119]

[9]. Seiler M, Huang CC, Szalma S, Bhanot G. ConsensusCluster: a software tool for unsupervised cluster discovery in numerical data. Omics. 2010; 14:109-13. [PubMed: 20141333]

[10]. TCGA Data Portal Overview. The Cancer Genome Atlas project Web site. https://tcgadata.nci.nih.gov/tcga/

[11]. Network TCGA. Integrative analysis of genomic and molecular alterations in clear cell renal cell carcinoma. Nature. 2013

[12]. Geiss GK, Bumgarner RE, Birditt B, et al. Direct multiplexed measurement of gene expression with color-coded probe pairs. Nat Biotechnol. 2008; 26:317-25. [PubMed: 18278033]

[13]. Tibshirani R, Hastie T, Narasimhan B, Chu G. Diagnosis of multiple cancer types by shrunken centroids of gene expression. Proc Natl Acad Sci U S A. 2002; 99:6567-72. [PubMed: 12011421]

[14]. Parker JS, Mullins M, Cheang MC, et al. Supervised risk predictor of breast cancer based on intrinsic subtypes. J Clin Oncol. 2009; 27:1160-7. [PubMed: 19204204]

[15]. Zisman A. Risk group assessment and clinical outcome algorithm to predict the natural history of patients with surgically resected renal cell carcinoma. J Clin Oncol. 2002; 20:4559-66. [PubMed: 12454113]

[16]. Zigeuner R, Hutterer G, Chromecki T, et al. External validation of the Mayo Clinic stage, size, grade, and necrosis (SSIGN) score for clear-cell renal cell carcinoma in a single European centre applying routine pathology. Eur Urol. 2010; 57:102-9. [PubMed: 19062157]

[17]. Adamy A, Chong KT, Chade D, et al. Clinical characteristics and outcomes of patients with recurrence 5 years after nephrectomy for localized renal cell carcinoma. J Urol. 2011; 185:433-8. [PubMed: 21167521]

[18]. Kroeger N, Choueiri TK, Lee JL, et al. Survival outcome and treatment response of patients with late relapse from renal cell carcinoma in the era of targeted therapy. Eur Urol. In press. DOI: 10.1016/j.eururo.2013.07.031.

[19]. Gerlinger M, Rowan AJ, Horswell S, et al. Intratumor heterogeneity and branched evolution revealed by multiregion sequencing. N Engl J Med. 2012; 366:883-92. [PubMed: 22397650]

[20]. Ellis MJ, Perou CM. The genomic landscape of breast cancer as a therapeutic roadmap. Cancer Discov. 2013; 3:27-34. [PubMed: 23319768]

[21]. Cancer Genoma Atlas Network. Comprehensive molecular portraits of human breast tumours. Nature. 2012; 490:61-70. [PubMed: 23000897]

[22]. Puvvada S, Kendrick S, Rimsza L. Molecular classification, pathway addiction, and therapeutic targeting in diffuse large B cell lymphoma. Cancer Genet. 2013; 206:257-65. [PubMed: 24080457]

[23]. Sadanandam A, Lyssiotis CA, Homicsko K, et al. A colorectal cancer classification system that associates cellular phenotype and responses to therapy. Nat Med. 2013; 19:619-25. [PubMed: 23584089]

[24]. De Sousa EMF, Wang X, Jansen M, et al. Poor-prognosis colon cancer is defined by a molecularly distinct subtype and develops from serrated precursor lesions. Nat Med. 2013; 19:614-8. [PubMed: 23584090]

[25]. Rini BI, Zhou M, Aydin H, et al. Identification of prognostic genomic markers in patients with localized clear cell renal cell carcinoma (ccRCC) [abstract 4501]. J Clin Oncol. 2010; 28(Suppl) 


\section{Take-home message}

A 34-gene subtype predictor effectively classifies clear cell renal cell carcinoma tumors according to $\mathrm{ccA}$ or ccB subtype. The predictor can be used for future analyses involving risk for developing metastatic disease and cancer-specific outcomes. 


\section{5 clear cell tumors}

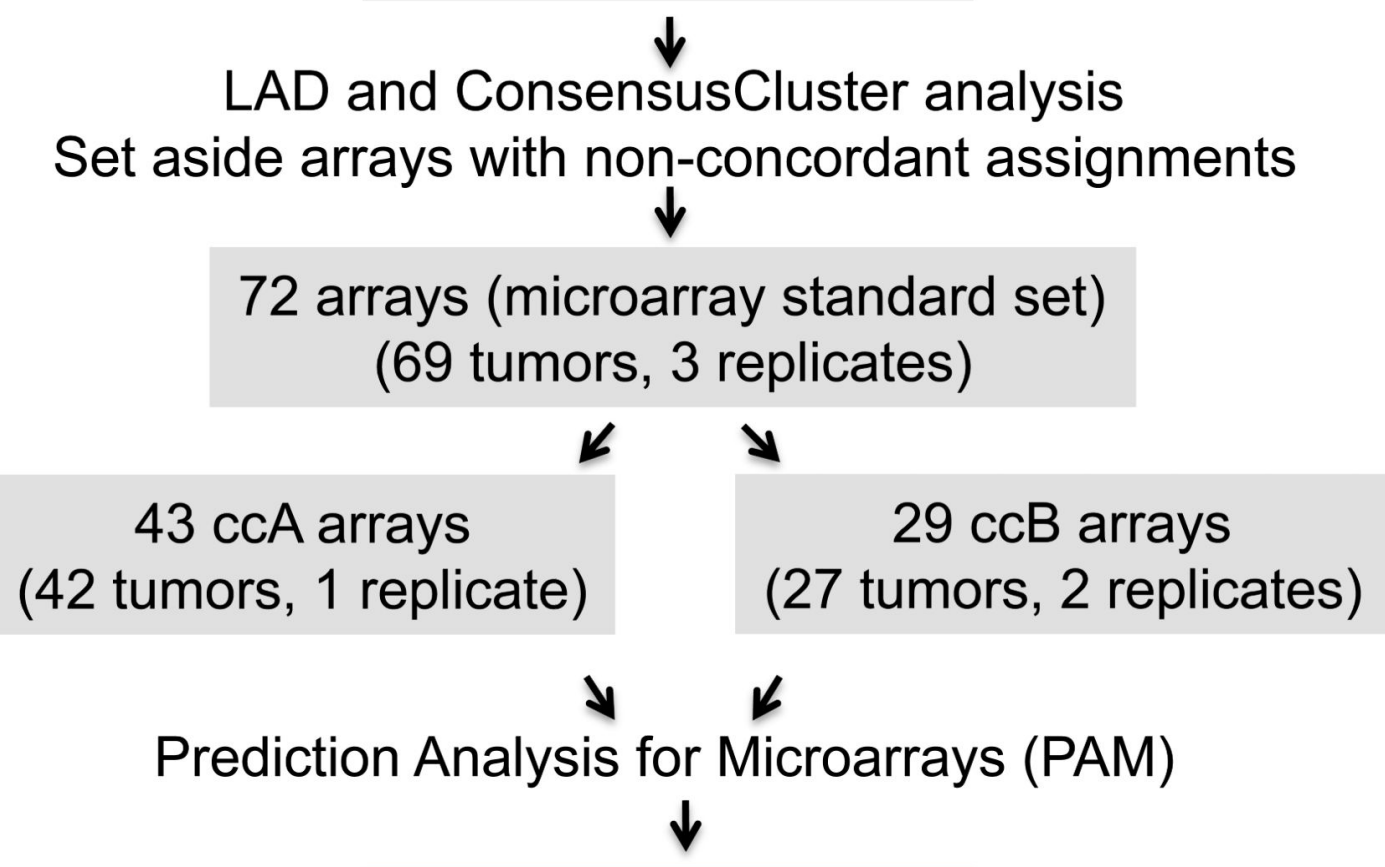

\section{Predictive biomarkers: \\ ClearCode34}

Fig. 1.

Workflow for biomarker discovery: steps taken to identify the 34 genes that classify ccA and ccB tumors.

$\mathrm{LAD}=$ logical analysis of data. 


\section{ClearCode34}

$\downarrow$

Analyze ClearCode34 in TCGA data $(\mathrm{N}=380)$

$\downarrow$

Validate ClearCode34 in UNC cohort $(\mathrm{N}=156)$

Combine TCGA and UNC cohorts $(\mathrm{N}=530)$

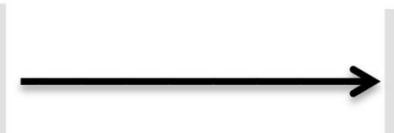

$\downarrow$

Randomly split combined cohort into training and test set to develop a tumor relapse risk model information

\section{Compare model to} established algorithms

Fig. 2.

Order of analyses to develop and validate the relapse risk model. Diagram of analyses to validate the efficiency of the biomarkers to classify tumors and predict prognostic outcomes. TCGA = the Cancer Genome Atlas; UNC = University of North Carolina. 
A.

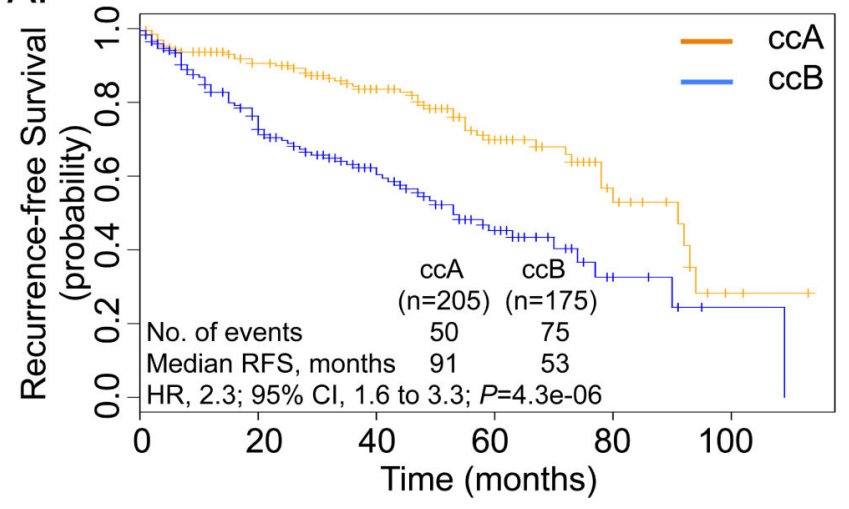

C.

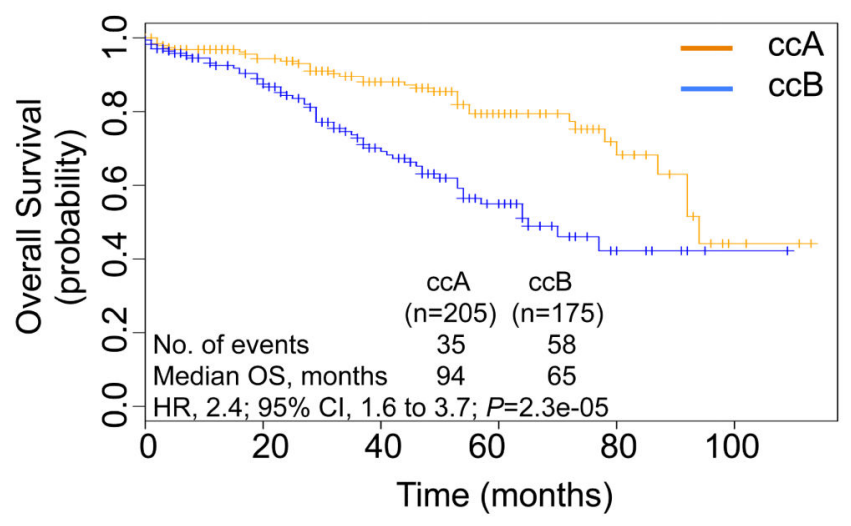

B.

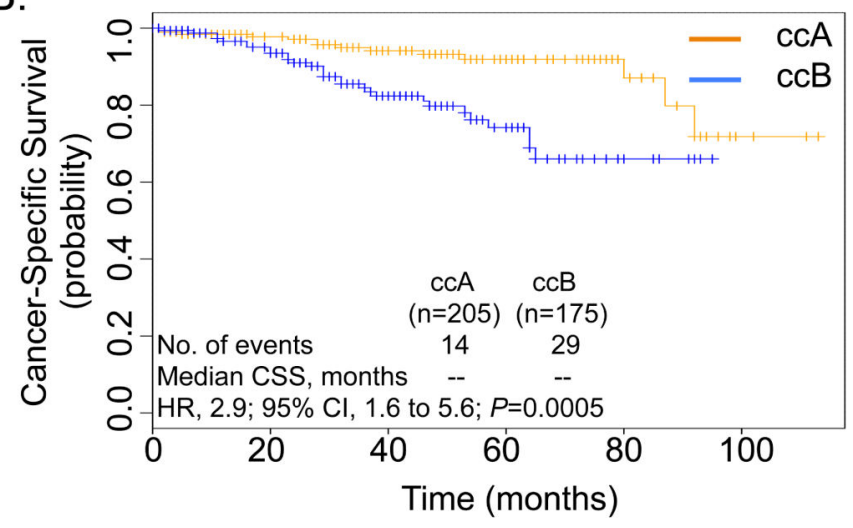

Fig. 3.

Tumor classification from the Cancer Genome Atlas (TCGA) shows distinct prognostic outcomes. Prediction analysis for microarray classified 380 untreated, nonmetastatic clear cell renal cell carcinoma tumors from TCGA as either $\mathrm{ccA}$ or $\mathrm{ccB}$, using the 34-gene classifier, ClearCode34. Kaplan-Meier curves were used to calculate (a) recurrence-free survival (RFS), (b) cancer-specific survival (CSS), and(c) overall survival (OS) for ccA and ccB patients. ccB-typed patients had a median RFS and OS of 53 and 65 mo, respectively, while patients with ccA-typed tumors had a 50\% survival probability of 91 and 94 mo for RFS and OS, respectively.

$\mathrm{HR}=$ hazard ratio; $\mathrm{CI}=$ confidence interval. 
A.
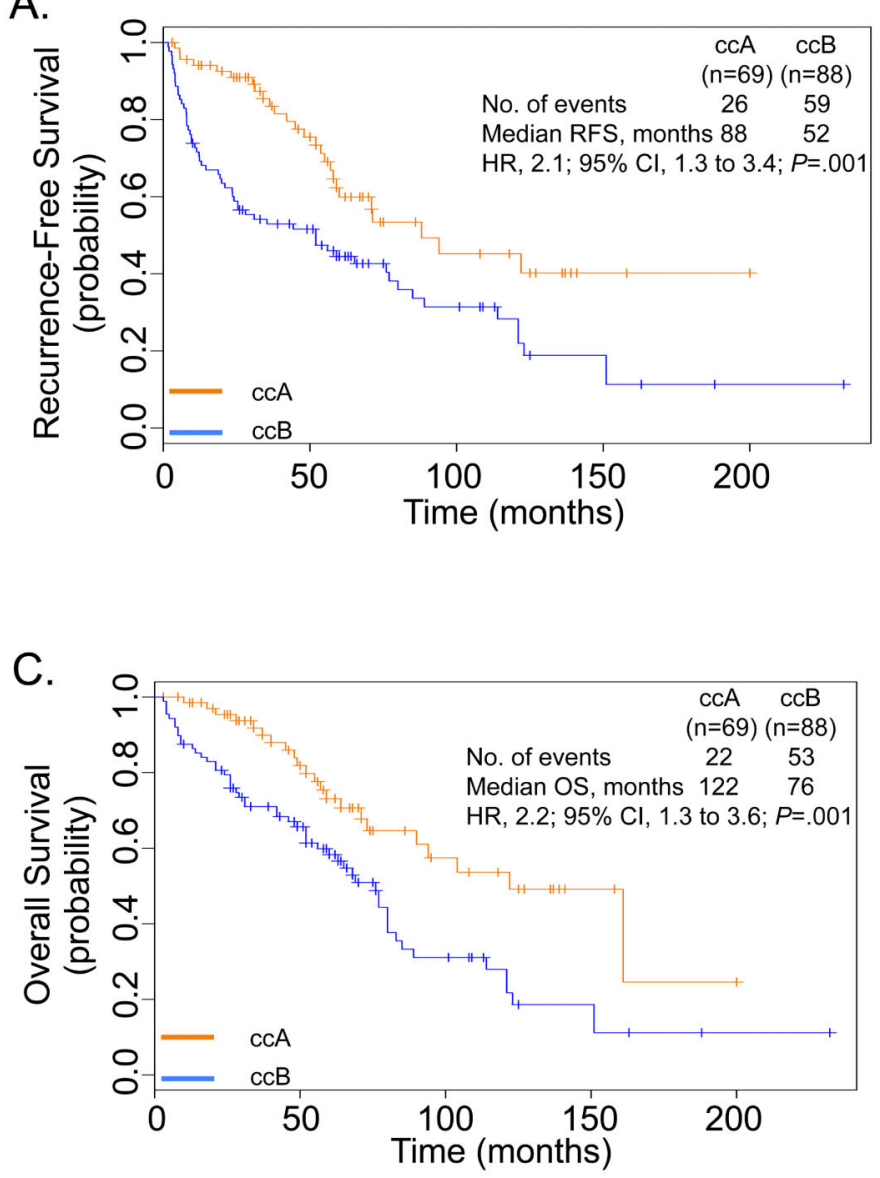

B.

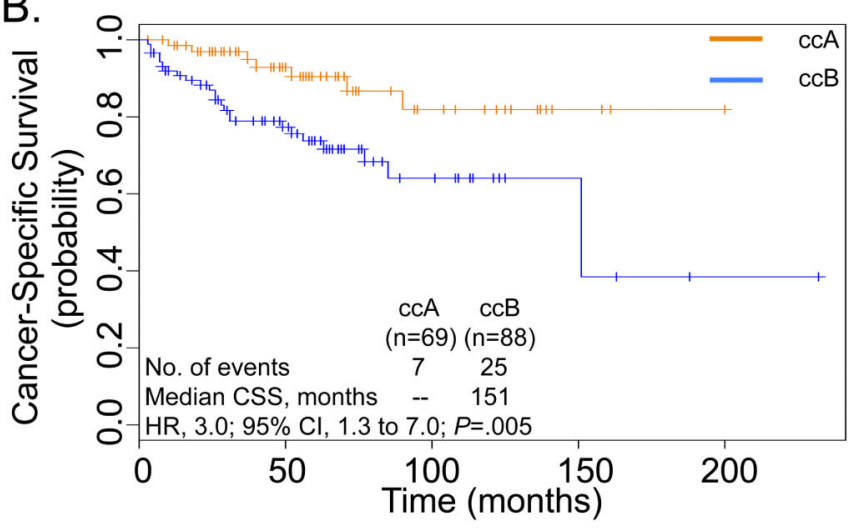

Fig. 4.

Clear cell renal cell carcinoma (ccRCC) classifier recapitulates survival outcomes for subtypes in clinical cohort. Whole lysates from 157 nonmetastatic, archived ccRCC primary tumor samples were subjected to NanoString gene expression analysis (NanoString Technologies Inc, Seattle, WA, USA). Kaplan-Meier plots of the independent cohort using ClearCode34 show ccB patients have significantly lower probabilities of (a) recurrence-free survival (RFS), (b) cancer-specific survival (CSS), and (c) overall survival (OS) compared to $c c \mathrm{~A}$.

$\mathrm{HR}=$ hazard ratio $; \mathrm{CI}=$ confidence interval. 

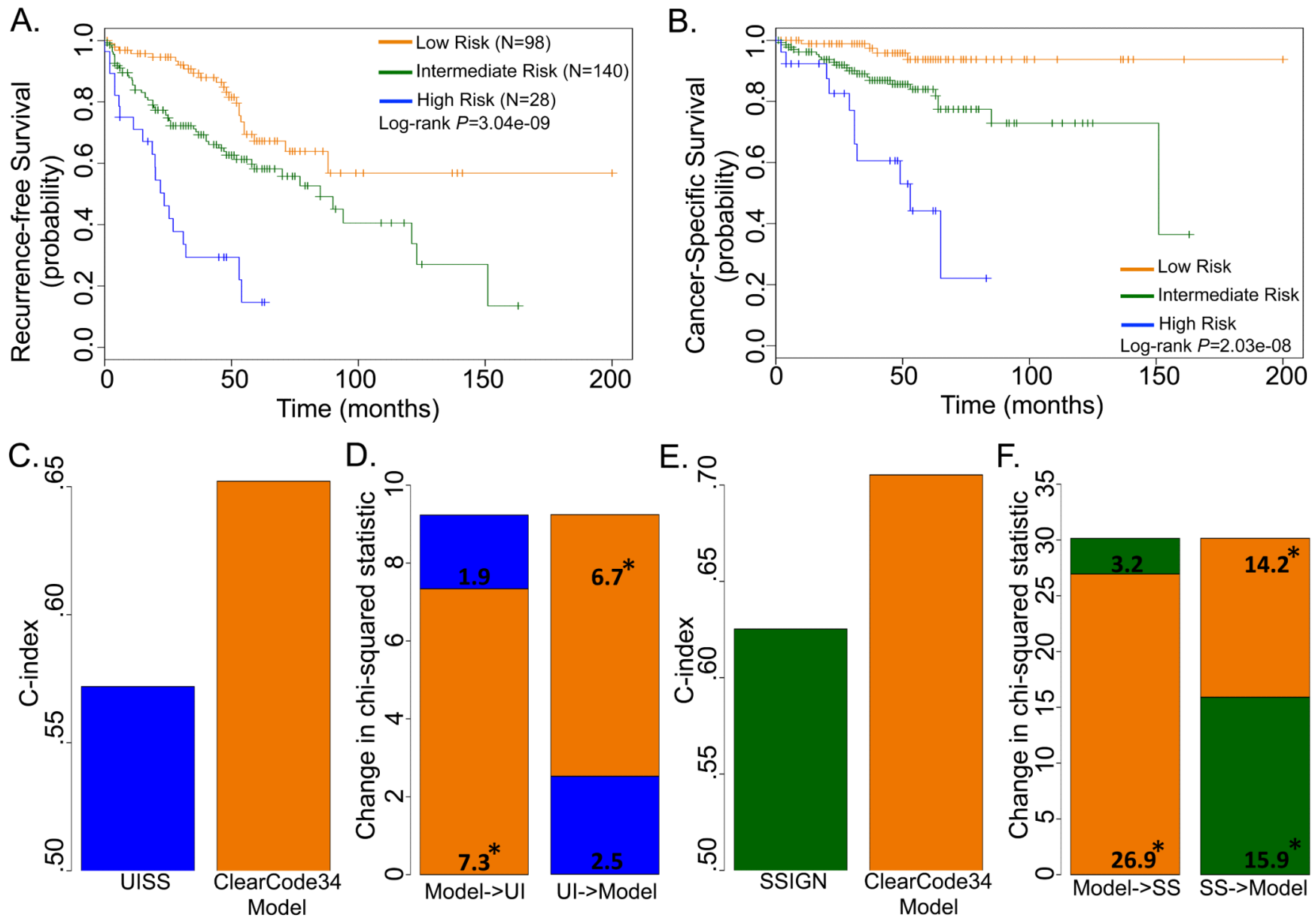

Fig. 5.

ClearCode 34 prognostic model can evaluate patient risk. A randomized training set of 265 patients from the Cancer Genome Atlas (TCGA) project and clinical cohorts were used to train a model to identify low-, intermediate-, and high-risk groups for tumor recurrence using clear cell renal cell carcinoma (ccRCC) subtype status ( $\mathrm{ccA} / \mathrm{ccB})$, tumor stage, and histologic Fuhrman grade. The model was applied to the test set $(n=266)$ to predict (a) recurrence and (b) cancer-specific death, revealing a highly significant risk profile integrating clinical and biologic features. (c and e) Co-occurrence index (C-index) and (d and f) multivariate analysis validated the efficacy of the model using the three risk groups to predict risk of ccRCC death over the established algorithms University of California, Los Angeles Integrated Staging System (UISS) and Mayo Clinic Stage, Size, Grade, and Necrosis (SSIGN) score. Chi-square statistic values resulting from multivariate regression depict the additive value of the three risk models.

$* p<0.05$. 
Table 1

\section{Expression of ClearCode34}

\begin{tabular}{|c|c|}
\hline Gene & ccRCC subtype \\
\hline MAPT & $\operatorname{ccA}$ \\
\hline STK32B & $\mathrm{ccA}$ \\
\hline FZD1 & $\mathrm{ccA}$ \\
\hline RGS5 & $\operatorname{ccA}$ \\
\hline GIPC2 & $\mathrm{ccA}$ \\
\hline PDGFD & $\operatorname{ccA}$ \\
\hline EPAS1 & $\operatorname{ccA}$ \\
\hline MAOB & $\operatorname{ccA}$ \\
\hline CDH5 & $\mathrm{ccA}$ \\
\hline TCEA3 & $\operatorname{ccA}$ \\
\hline LEPROTL1 & $\mathrm{ccA}$ \\
\hline BNIP3L & $\mathrm{ccA}$ \\
\hline EHBP1 & $\operatorname{ccA}$ \\
\hline VCAM1 & $\mathrm{ccA}$ \\
\hline PHYH & $\operatorname{ccA}$ \\
\hline PRKAA2 & $\mathrm{ccA}$ \\
\hline SLC4A4 & $\operatorname{ccA}$ \\
\hline ESD & $\mathrm{ccA}$ \\
\hline TLR3 & $\mathrm{ccA}$ \\
\hline NRP1 & $\mathrm{ccA}$ \\
\hline C11orf1 & $\mathrm{ccA}$ \\
\hline ST13 & $\mathrm{ccA}$ \\
\hline ARNT & $\mathrm{ccA}$ \\
\hline C13orf1 & $\mathrm{ccA}$ \\
\hline SERPINA3 & $\mathrm{ccB}$ \\
\hline SLC4A3 & $\mathrm{ccB}$ \\
\hline MOXD1 & $\mathrm{ccB}$ \\
\hline KCNN4 & $\mathrm{ccB}$ \\
\hline ROR2 & $\mathrm{ccB}$ \\
\hline FLJ23867 & $\mathrm{ccB}$ \\
\hline FOXM1 & $\mathrm{ccB}$ \\
\hline UNG2 & $\mathrm{ccB}$ \\
\hline GALNT10 & $\mathrm{ccB}$ \\
\hline GALNT4 & $\mathrm{ccB}$ \\
\hline
\end{tabular}

ccRCC $=$ clear cell renal cell carcinoma. 
Table 2

Patient demographics and clinical characteristics of The Cancer Genome Atlas cohort

\begin{tabular}{|l|l|l|}
\hline Characteristic & No. & \% \\
\hline Sex & & \\
\hline Male & 243 & 64 \\
\hline Female & 137 & 36 \\
\hline Age & & \\
\hline Median & 61 & \\
\hline Range & $29-90$ & \\
\hline Ethnicity & & \\
\hline White & 330 & 87 \\
\hline Black & 17 & 5 \\
\hline Hispanic & 20 & 5 \\
\hline Asian & 8 & 2 \\
\hline Unknown & 5 & 1 \\
\hline Fuhrman grade & & \\
\hline I & 6 & 2 \\
\hline II & 181 & 48 \\
\hline III & 150 & 39 \\
\hline IV & 37 & 10 \\
\hline Unknown & 6 & 1 \\
\hline Staging (TNM) & & \\
\hline I & 216 & 56 \\
\hline II & 45 & 12 \\
\hline III & 116 & 31 \\
\hline IV & 3 & 1 \\
\hline
\end{tabular}


Table 3

Patient demographics and clinical characteristics of the University of North Carolina cohort

\begin{tabular}{|l|l|l|}
\hline Characteristic & No. & \% \\
\hline Sex & & \\
\hline Male & 94 & 60 \\
\hline Female & 63 & 40 \\
\hline Age & & \\
\hline Median & 58 & \\
\hline Range & $19-82$ & \\
\hline Ethnicity & & \\
\hline White & 102 & 65 \\
\hline Black & 44 & 28 \\
\hline Hispanic & 5 & 3 \\
\hline Asian & 1 & 1 \\
\hline Native American & 5 & 3 \\
\hline Fuhrman grade group & & \\
\hline I & 3 & 2 \\
\hline II & 70 & 45 \\
\hline III & 70 & 45 \\
\hline IV & 14 & 8 \\
\hline Staging (TNM) & & \\
\hline I & 78 & 50 \\
\hline II & 23 & 14 \\
\hline III & 56 & 36 \\
\hline & & \\
\hline & & \\
\hline & & \\
\hline
\end{tabular}

